\title{
Dynamic light scattering near the isotropic-blue phase transition of cholesteryl-oleylcarbonate
}

\author{
by A. BÖTTGER, L. NIESSEN, J. COELINGH, D. FRENKEL \\ and R. J. J. ZIJLSTRA \\ Fysisch Laboratorium, Rijksuniversiteit Utrecht, P.O. Box 80.000, \\ 3508 TA Utrecht, The Netherlands
}

(Received 5 June 1987; accepted 7 December 1987)

\begin{abstract}
We report a light scattering study in the isotropic phase of a blue phaseforming fluid (cholesteryl-oleylcarbonate). An analysis of the intensity correlation function of the depolarized scattered light indicates that a two variable theory is not adequate to describe the intensity autocorrelation function. Our data are well-described by a stretched exponential decay of the collective orientational fluctuation. Such behaviour is usually observed in the vicinity of a glass transition. In addition we report measurements of the refractive index in the vicinity of the isotropic-blue phase transition.
\end{abstract}

\section{Introduction}

The phase transition from an isotropic liquid to an orientationally ordered fluid such as a nematic or cholesteric liquid crystal is usually weakly first order. As a consequence, the actual phase transformation is preceded by strong static and dynamic precursor effects [1]. In particular, the amplitude and the relaxation time of collective orientational fluctuations increase dramatically as the transition is approached, although the temperature at which both quantities actually diverge is never reached. The first order phase transition to the orientationally ordered phase takes place typically $1 \mathrm{~K}$ above that hypothetical point. A systematic light scattering study of collective orientational fluctuations in the vicinity of the isotropic-nematic transition is described in [2] and in the paper by Gierke and Flygare [3]. The latter paper clearly demonstrates that the critical slowing-down effect of collective orientational fluctuations in the vicinity of the isotropic-nematic transition is due almost exclusively to an increase in the static orientational correlation length; other transport properties, such as for instance the shear viscosity, exhibit hardly any pretransitional enhancement.

The situation is expected to be quite different for fluids that have a phase transition from the isotropic to the blue phase (BP) $[4,5]$ because there is a strong pretransitional increase of the shear viscosity as the transition to the blue phase is approached $[6,7]$; this was not the case with the isotropic-nematic transition studied in [2].

In this paper we report dynamic light scattering measurements of collective orientational fluctuations in the isotropic phase of cholesteryl-oleylcarbonate (COC). This material was studied previously by Mahler $e t$ al. [8] and by Harada and Crooker [9]; it is known to have at least one stable blue phase between the cholesteric phase and the isotropic phase $[10,11]$. In addition to performing the dynamic light scattering experiments, we have also measured the refractive index of $\mathrm{COC}$ over the temperature 
range of interest, together with the shear viscosity and the isobaric heat capacity, $C_{P}$. On the basis of the results of these measurements we shall argue that our dynamic light scattering data do not appear to be consistent with a two variable hydrodynamic theory [12]. Such a theory was applied successfully by Gierke and Flygare [3] and Stinson et al. [2] to the analysis of the light scattering results on MBBA. Other authors [9] employed it subsequently to analyse pretransitional light scattering in the vicinity of the isotropic to blue phase transition.

\section{Experimental}

\subsection{Sample preparation}

The COC used in our experiments was obtained from Duphar B.V. (Veenendaal, The Netherlands). The material was made dust-free by centrifugation for several hours at a temperature several degrees above $T_{\mathrm{BPI}}$. Before the light scattering experiments the samples were degassed under vacuum. The samples were then used without further purification. The purity and stability, checked by a thin layer chromatogram, showed no difference before and after the experiments. Although a small spot due to impurities was found, the material showed transition temperatures comparable with those found in the highly purified material. The transition from the cholesteric phase to an optically isotropic phase was observed at a temperature of $307 \cdot 4 \pm 0 \cdot 1 \mathrm{~K}$, the smecticcholesteric phase transition was found at $292.9 \pm 0 \cdot 1 \mathrm{~K}$; this is in agreement with [10].

D.S.C. measurements showed that a second phase transition takes place at $308.0 \mathrm{~K}$. This suggests that a blue phase is stable over $0.6 \mathrm{~K}$. Additional evidence for the presence of this blue phase comes from the refractive index measurements described later. Our shear viscosity measurements (in the isotropic phase) agree well with those reported by Yamada and Fukada [13].

\subsection{Refractive index}

The refractive indices of $\mathrm{COC}$ were studied using an Abbe refractometer. The prisms used had a refractive index $n_{p}=1 \cdot 7$. In order to obtain an accuracy of $10^{-4}$ in the refractive index it was necessary to reduce temperature fluctuations. A stability of $0.01 \mathrm{~K}$ was reached by placing the refractometer in a thermally insulated box. A Pt-resistor placed in the setting of the upper prism was used to measure the temperature. Measurements were done both upon heating and upon cooling the sample. The results for two different wavelengths, $\lambda_{0}=633 \mathrm{~nm}$ and $\lambda_{0}=589 \mathrm{~nm}$, are plotted in figures $1(a)$ and $1(b)$. The persistence of the isotropic refractive index, below the limit of stability of the cholesteric phase, provides additional evidence for the presence of a (metastable) blue phase in this temperature range. It is well-known that blue phases can be supercooled easily [14] but our measurements show that the range of metastability is exceptionally large.

\subsection{Light scattering}

The light scattering experiments were performed with the same set-up as that described in [15]. The set-up consists of a toluene filled temperature controlled bath illuminated by a krypton laser $\left(\lambda_{0}=647 \cdot 1 \mathrm{~nm}\right)$. The polarization of the incident beam was set perpendicular to the scattering plane. We studied the intensity as well as the intensity autocorrelation function of the depolarized component at a constant scattering angle, $\theta$ of $33^{\circ}$. 

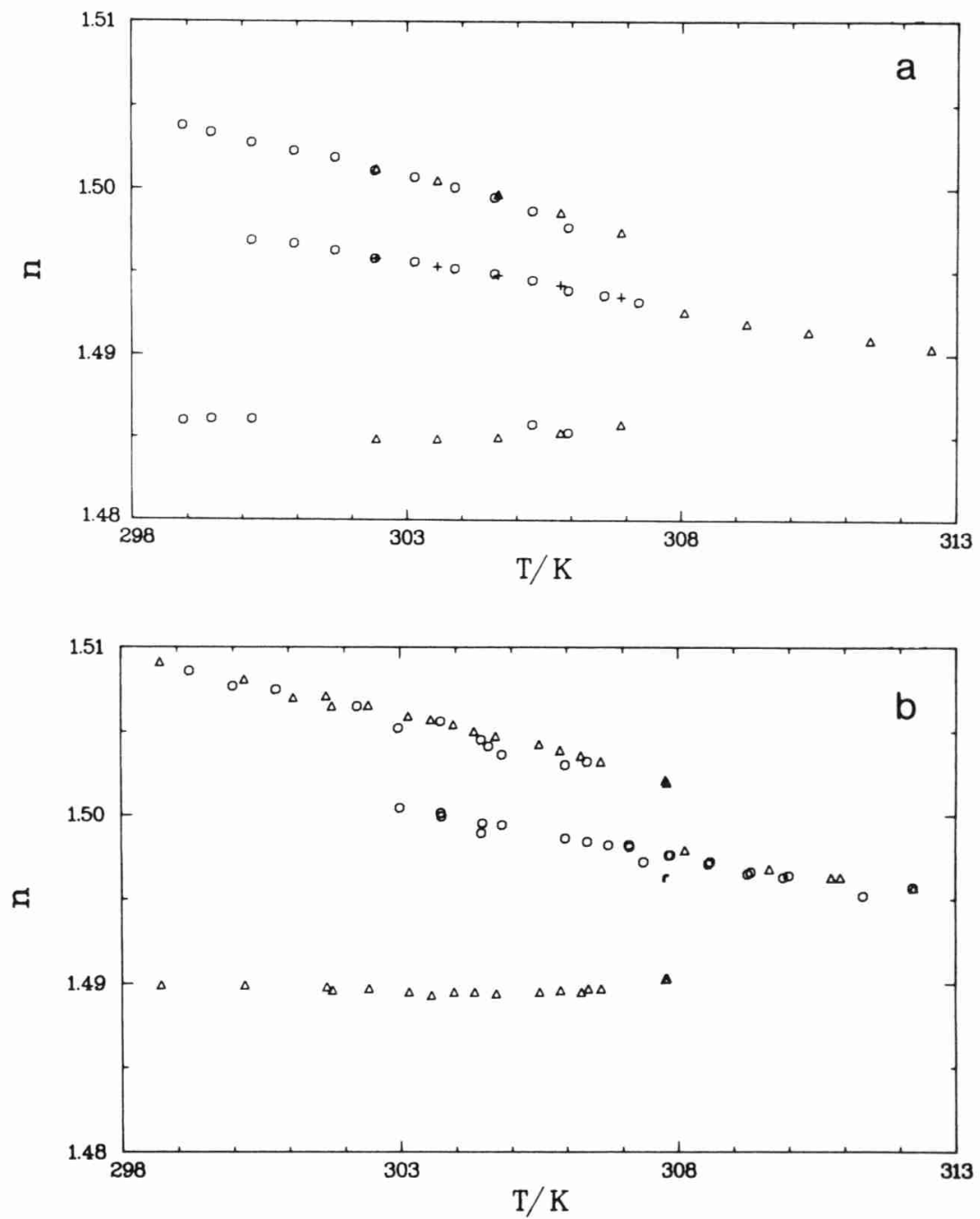

Figure 1. Refractive index of COC measured at $(a) \lambda=633 \mathrm{~nm}$ and $(b) \lambda=589 \mathrm{~nm}$; $\Delta$, decreasing temperature; $O$, increasing temperature; + , computed values $n=2 n_{0}+n_{\mathrm{e}} / 3$, note that $n_{0}>n_{\mathrm{e}}$.

\section{Results}

The intensity measurements were performed over a temperature range of $8 \mathrm{~K}$ above $T_{\mathrm{c}}$. The data were corrected for background contributions and laser fluctuations. Each time the temperature was lowered we monitored the time evolution of scattered intensity. The light scattering data were collected after the intensity had reached a stable value over, at least, 1 hour.

Figure 2 shows a plot of the temperature dependence of the intensity of the depolarized component of the scattered light as a function of temperature. If we assume that the light scattering intensity is due exclusively to collective fluctuations of the molecular orientations, then the Landau-de Gennes theory [18] predicts that the temperature dependence of the scattered intensity should be 
given by

$$
\begin{aligned}
I_{\mathrm{VH}}= & C \frac{k T}{\left(T-T^{*}\right)}\left\{\frac{\left(1+\xi_{2}^{2} q^{2}\right)}{\left(1+\xi_{2}^{2} q^{2}\right)^{2}-q_{0}^{2} q^{2} \xi_{1}^{4}} \cos ^{2} \frac{\theta}{2}\right. \\
& \left.+\frac{\left(1+\xi_{1}^{2} q^{2}\right)}{\left(1+\xi_{1}^{2} q^{2}\right)^{2}-4 q_{0}^{2} q^{2} \xi_{1}^{4}} \sin ^{2} \frac{\theta}{2}\right\},
\end{aligned}
$$

where $k$ is the Boltzmann constant, $q_{0}=\pi / p$ with $p$ as the cholesteric pitch, $\xi_{1,2}$ are the correlation lengths

$$
\xi_{1}=\frac{L_{1}}{A}, \quad \xi_{2}=\frac{L_{1}+L_{2}}{A}, \quad q=\frac{4 \pi}{\lambda_{0}} n \sin \frac{\theta}{2},
$$

and $C$ is a constant. As can be seen from figure 2, a plot of $T / I$ versus temperature appears to follow the linear temperature dependence predicted by the Landaude Gennes theory, except in the immediate vicinity of the phase transition. A linear least-squares fit to the data points in figure 2 yields the estimate that $T^{*}=306 \cdot 71 \pm 0.007 \mathrm{~K}$.

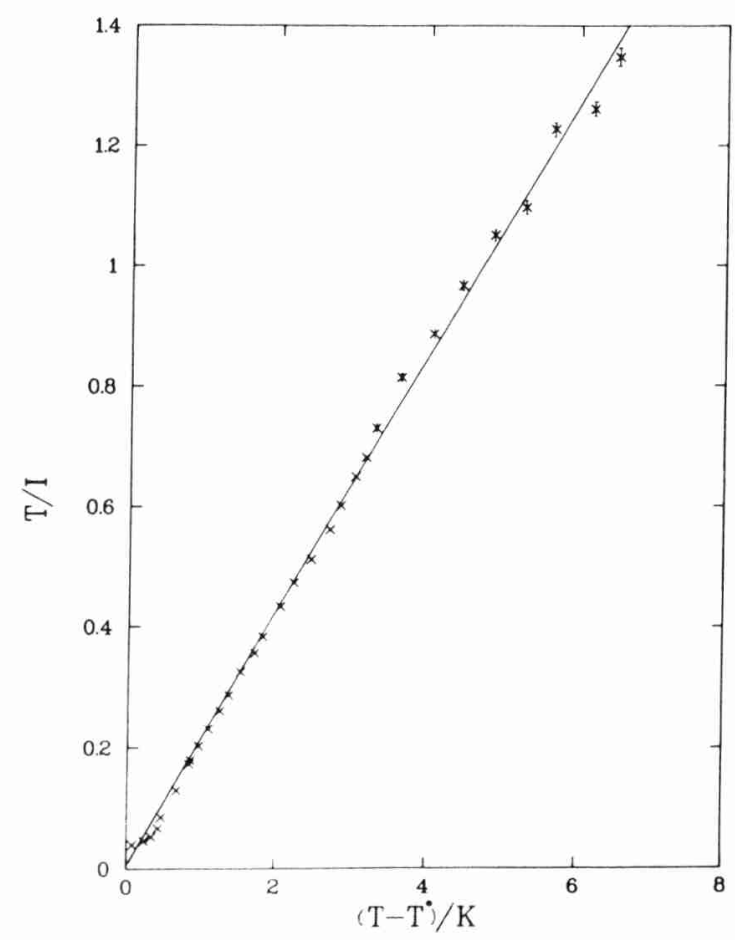

Figure 2. $T / I$ data in arbitrary units of COC versus temperature.

In addition to the measurements of the static light scattering of the COC in the isotropic phase, we employed photon correlation spectroscopy to study the dynamics of the collective orientational fluctuations above the I-BP transition. The dynamic light scattering was measured using a 64-channel Malvern K-7026 single-clipped correlator with a minimum channel width of $20 \times 10^{-9} \mathrm{~s}$. As the relaxation times were found to depend strongly on temperature we checked carefully for possible heating effects caused by the incident laser beam. Near the transition temperature we 


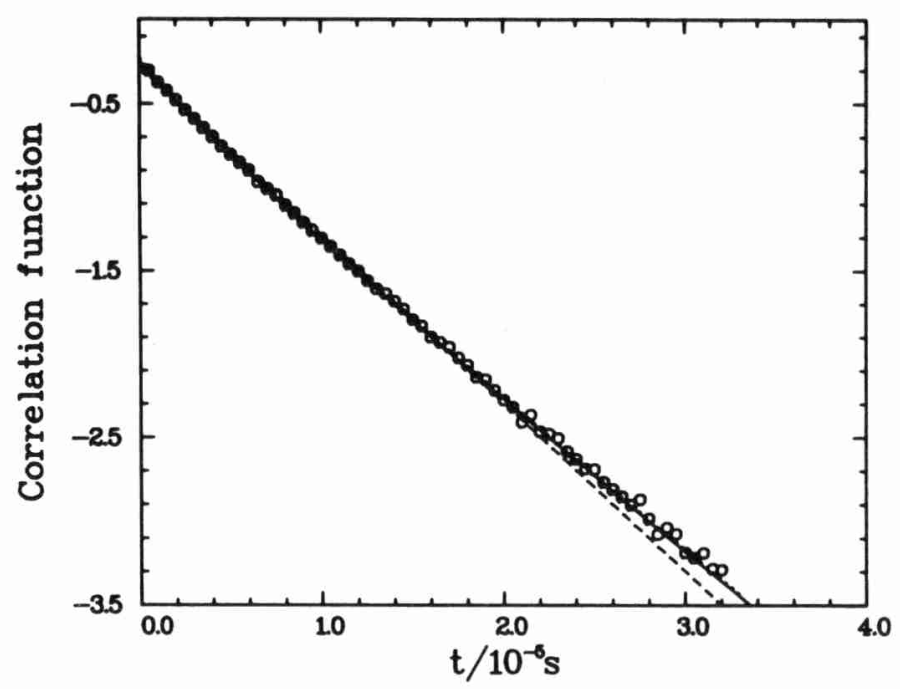

Figure 3. An example of a typical fit of the correlation function to one exponential (- - $)$, the sum of two exponentials $(-)$ and a stretched exponential (----). The figure clearly shows that the fit to one exponential does not describe the correlation function in the whole range of time. The correlation function was measured at $T=309 \cdot 25 \mathrm{~K}$.

did observe a slight decrease in the measured correlation time when the laser power, $P$, was increased from 10 to $300 \mathrm{~mW}$. For this reason all results quoted subsequently are based on an extrapolation of the measured correlation times to laser power $P=0$.

Earlier light scattering experiments on COC and closely related compounds $[8,9,16,17]$ have indicated that the light scattering spectrum in the isotropic phase consists of a single lorentzian, whereas the spectrum in the blue phase is composed of two lorentzians. We have attempted to fit our data of the $\mathrm{VH}$ spectrum in the isotropic phase of COC by a single lorentzian, which in the time domain corresponds to fitting the intensity correlation function to a single exponential. However, we found that even in the isotropic phase a single exponential fitted poorly to our data. A typical value of the reduced $\chi^{2}$ for a fit to one exponential was 6 whereas for a fit to a sum of exponentials it was 1 (see figure 3 ). Actually this is not surprising because the two variable hydrodynamic theory $[12,18,19]$ that describes the VV and VH light scattering spectra for cylindrically symmetric molecules predict that in the limit $q^{2} \eta / \Gamma \gg 1$, where $\eta$ is the shear viscosity, the VH spectrum should consist of two lorentzians instead of one;

$$
I_{\mathrm{VH}}=\frac{\Gamma}{\omega^{2}+\Gamma^{2}} \sin ^{2} \frac{\theta}{2}+\frac{\Gamma /(1-R)}{\omega^{2}+\Gamma^{2} /(1-R)^{2}} \frac{1}{2} \cos ^{2} \frac{\theta}{2},
$$

and the VV spectrum consists of a single lorentzian

$$
I_{\mathrm{VV}}=\frac{\Gamma}{\omega^{2}+\Gamma^{2}}
$$

where $R$ is a parameter that measures the coupling strength between molecular rotation and the transverse velocity field. In [9], Harada and Crooker obtain $R$ by comparing the effective linewidths of the VV and VH spectra assuming that the latter 
is dominated by the second term on the right hand side of equation (1), and neglect the contribution of the density fluctuations [20] to the VV spectrum. The value for $R$ obtained in [9] is $0 \cdot 2$ and it is almost independent of temperature. As can be seen from equation (1), $R$ can also be determined from a measurement of the two independent components of the $\mathrm{VH}$ spectrum. We chose to determine $R$ in this way. To this end we fitted the $\mathrm{VH}$ electric field correlation function to a sum of two exponentials. The resulting values for the relaxation rates $\Gamma$ are shown in figure 4 . The value of $R$ deduced from the ratio of the relaxation rates of the two exponents is shown in figure 4 . Note that $R$ in figure 5 typically has values between 0.6 and 0.8 which is significantly larger than $0 \cdot 2$.

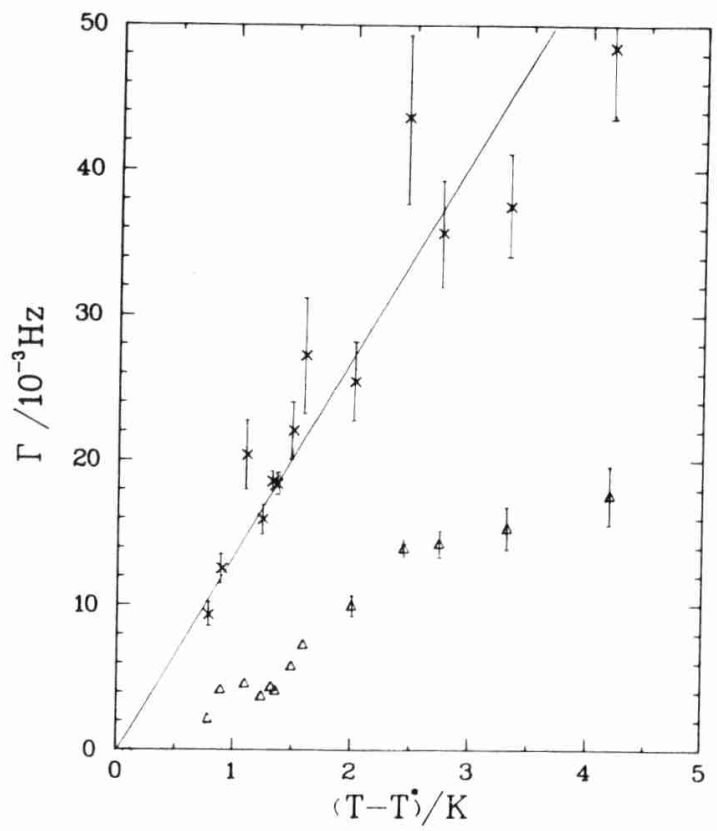

Figure 4. Effective linewidths $\Gamma$ of the VH spectrum (cf. equation (1)).

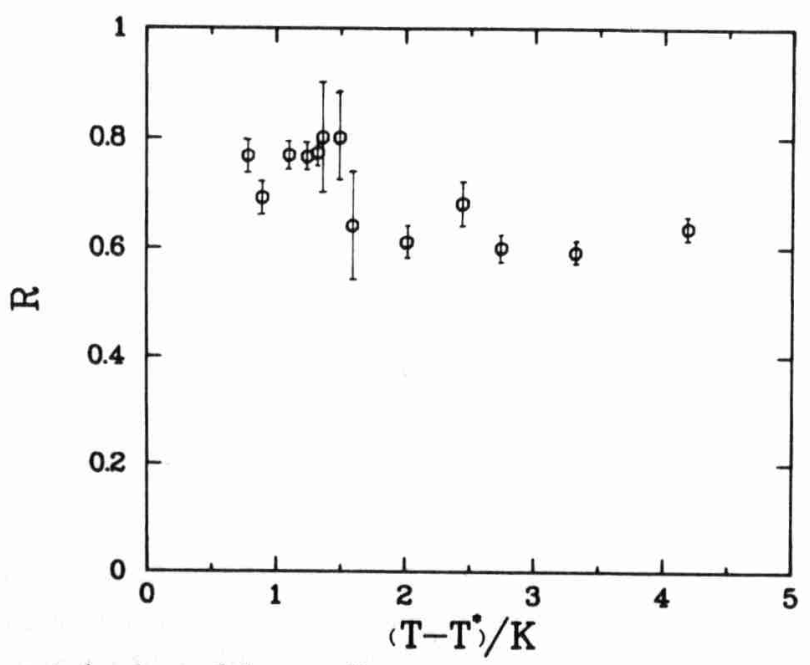

Figure 5. Computed values of the coupling parameter $R$ obtained from the effective linewidths (VH spectrum). 
The values of $\beta$ and $\tau$ obtained from a two parameter fit of the normalized correlation function.

\begin{tabular}{|c|c|c|}
\hline$T / K$ & $\beta$ & $\bar{\tau} / 10^{-5} \mathrm{~s}$ \\
\hline $311 \cdot 00$ & $0.796 \pm 0.010$ & $1.089 \pm 0.015$ \\
\hline $310 \cdot 84$ & $0.878 \pm 0.007$ & $1.423 \pm 0.008$ \\
\hline $309 \cdot 53$ & $0.891 \pm 0.006$ & $1.564 \pm 0.017$ \\
\hline $309 \cdot 24$ & $0.912 \pm 0.005$ & $1.927 \pm 0.074$ \\
\hline $308 \cdot 82$ & $0.896 \pm 0.008$ & $2.540 \pm 0.020$ \\
\hline $308 \cdot 40$ & $0.901 \pm 0.005$ & $3.406 \pm 0.030$ \\
\hline $308 \cdot 30$ & $0.910 \pm 0.005$ & $5.020 \pm 0.528$ \\
\hline $307 \cdot 91$ & $0.903 \pm 0.018$ & $6 \cdot 370 \pm 0.120$ \\
\hline $307 \cdot 81$ & $0.902 \pm 0.005$ & $7 \cdot 130 \pm 0 \cdot 200$ \\
\hline $307 \cdot 70$ & $0.901 \pm 0.004$ & $8.450 \pm 0.049$ \\
\hline $307 \cdot 59$ & $0.883 \pm 0.005$ & $12 \cdot 110 \pm 0 \cdot 020$ \\
\hline $307 \cdot 48$ & $0.870 \pm 0.008$ & $14.500 \pm 0.087$ \\
\hline $306 \cdot 01$ & $0.897 \pm 0.007$ & $40.080 \pm 1.000$ \\
\hline
\end{tabular}

This discrepancy in the values of $R$ (e.g. those obtained by comparison of $I_{\mathrm{Vv}}$ and $I_{\mathrm{VH}}$ and those obtained by the measurement of the linewidths) constitutes a strong indication that the simple two variable hydrodynamic theory is not adequate to describe the VH spectrum in the isotropic phase of COC. Actually, this is not surprising. It is well known that the light scattering spectrum of viscous liquids is often better described by a three variable hydrodynamic theory [21, 22]. However, such three variable theories contain more adjustable parameters than we can hope to determine with the present experiments, even if we ignore the complexities introduced by the fact that COC consists of chiral molecules. But actually there is a good reason for not using three or more variable theories to describe our data, namely that in strongly interacting liquids the relaxation of fluctuations often follows a stretched exponential form $[23,24]$. Our measured normalized correlation functions fit, within the estimated statistical error, to this simple two parameter function $f(t ; \beta, \bar{\tau})$;

$$
f(t ; \beta, \bar{\tau})=\exp \left(-(\tau / \bar{\tau})^{\beta}\right) .
$$

The resulting values for $\beta$ and $\bar{\tau}$ have been collected in the table. The values for the reduced $\chi^{2}$ of the present fits are at least as good as those obtained in the fits to a sum of exponentials (cf. figure 3). But the latter fit involves three instead of two adjustable parameters. Stretched exponential decay of fluctuations is often found in strongly interacting liquids [25], in particular in the vicinity of the glass transition. Although the isotropic to blue phase transition is different from a glass transition, there is one important analogy, namely that the shear viscosity diverges as the transition is approached (we recall that such behaviour is not observed near the isotropic-nematic transition). The model of subsequently relaxing quantities, used by Palmer and Stein [24] leads to a Vogel-Fulcher law of the relaxation times, which is often observed in experiments in the vicinity of a glass transition. If we fit the relaxation time of collective orientational fluctuations to a Vogel-Fulcher law,

$$
\bar{\tau}=b \exp \left(a /\left(T-T_{0}\right)\right)
$$

$[26,27]$ (see Figure 6), we obtain a value for $T_{0}$ of $306.4 \pm 0.05 \mathrm{~K}$. This temperature is in the range of stability of the blue phase.

The present analysis of the VH light-scattering spectrum of COC in the isotropic phase in terms of a stretched exponential correlation function is rather speculative and 


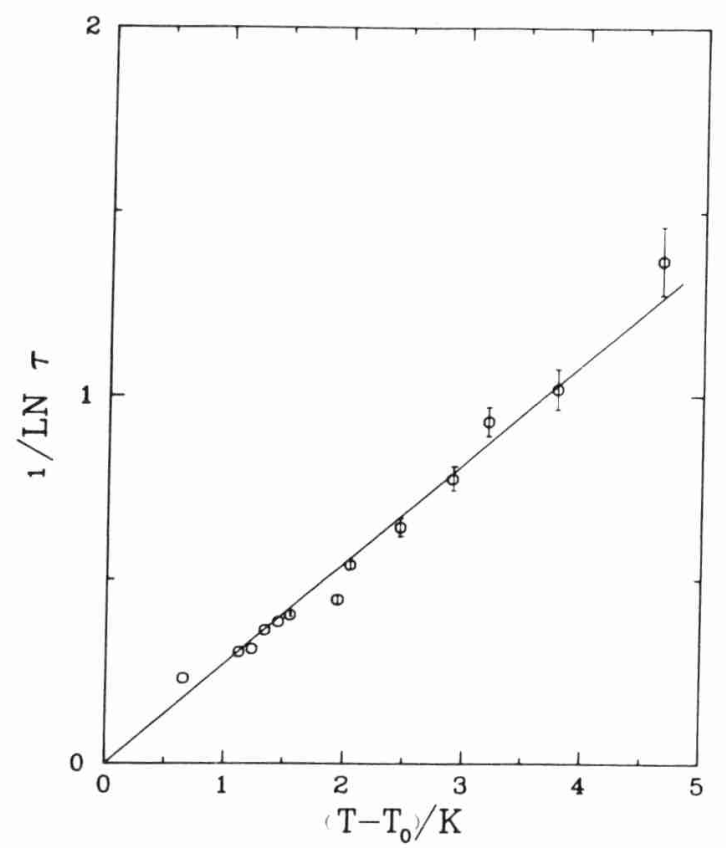

Figure 6. Values of $1 / \ln \tau$, with $\tau=\bar{\tau} / b$ obtained from a fit of the electric field correlation function to a stretched exponential (cf. equation (2)).

independent confirmation by other techniques is certainly desirable. Nevertheless, it seems clear that the dynamics of pretransitional fluctuations near the I-BP transition cannot be described by the type of two variable hydrodynarnic theory that explains the dynamics of collective orientational fluctuations near the isotropic-nematic transition. We believe that the analogy between the approach to the blue phase transition and the glass transition is significant and interesting. It should be recalled that the transition to the blue phase is, in essence, a freezing transition. Blue phases I and II can be thought of as cubic lattices of disclinations. However, this freezing transition is very different from the freezing of simple liquids. For one thing, in a normal, isotropic three-dimensional liquid there are no pretransitional precursor effects to freezing. One reason is that the local packing in a liquid close to freezing is very different from the structure of the solid. As a consequence, there is an appreciable free energy barrier to crystal nucleation. However, no such local packing effects apply to the structure of an isotropic fluid while the transition to the blue phase is approached. In fact, local orientational ordering similar to that in the blue phase is expected to extend over a correlation length $\xi$ [28], which grows rapidly as the I-BP transition is approached. Hence, just as with the glass transition, the vicinity of the crystalline blue phase manifests itself through a dramatic increase of the relaxation time of those hydrodynamic modes that are frozen out in the low temperature phase, i.e. the transverse momentum modes and the collective reorientation. In contrast, it is only the latter mode which is affected in the vicinity of the isotropic-nematic transition.

This work was performed as a part of the research programme of the Stichting voor Fundamenteel Onderzoek der Materie (FOM) with financial support from the Nederlandse Organisatie voor Zuiver Wetenschappelijk Onderzoek (ZWO). 


\section{References}

[1] DE Gennes, P. G., 1974, Physics of Liquid Crystals (Clarendon Press).

[2] Stinson, T. W., Litster, J. D., and Clark, N. A., 1972, J. Phys., Paris, 33, C1-69.

[3] Gierke, T. D., and Flygare, W. H., 1974, J. chem. Phys., 61, 2231.

[4] Stegemeyer, H., and Bergmann, K., 1980, Liquid Crystals of One- and Two-Dimensional Order, edited by W. Helfrich and G. Heppke (Springer).

[5] Grebel, H., Hornreich, R. M., and Shtrikman, S., 1988, Phys. Rev. A, 28, 1114.

[6] Stegemeyer, H., and Pollmann, P., 1982, Molec. Crystals liq. Crystals, 82, 123.

[7] Keyes, P. H., and Ajgaonkar, D. B., 1977, Phys. Lett. A, 64, 298.

[8] Mahler, D. S., Keyes, P. H., and Daniels, W. B., 1975, Phys. Rev. Lett., 36, 491.

[9] Harada, T., and Crooker, P. P., 1975, Phys. Rev. Lett., 34, 1259.

[10] Gray, G. W., and Hannat, M., 1979, Molec. Crystals liq. Crystals, 53, 263.

[11] Nakahara, M., Yoshimura, Y., and Osugi, J., 1981, Bull. chem. Soc. Japan, 54, 99.

[12] Berne, B. J., and Pecora, R., 1976, Dynamic Light Scattering (J. Wiley).

[13] Yamada, T., and Fukada, E., 1973, Jap. J. appl. Phys., 12, 68.

[14] Meiboom, S., and Sammon, M., 1981, Phys. Rev. A, 24, 468.

[15] Böttger, A., Frenkel, D., van De Riet, E., and Zülstra, R. J. J., 1987, Liq. Crystals, 2, 539 .

[16] Keyes, P. H., and YAng, C. C., 1979, J. Phys., Paris, 40, C3-376.

[17] YanG, C. C., 1972, Phys. Rev. Lett., 28, 955.

[18] DE Gennes, P. G., 1971, Molec. Crystals. liq. Crystals, 12, 193.

[19] Shyr-Jin, T., and Kivelson, D., 1975, Molec. Phys., 29, 1.

[20] Schindler, M. E., and Crooker, P. P., 1979, Molec. Crystals liq. Crystals Lett., 49, 307.

[21] Chappell, P. J., et al., 1981, J. chem. Phys., 74, 5929. Allen, M. P., Chappell, P. J., and Kivelson, D., 1981, J. chem. Phys., 74, 5942. MacPhail, R. A., and Kivelson, D., 1984, J. chem. Phys., 80, 2102.

[22] Viovy, J. L., Searby, G. M., Fried, F., Vellutini, M. J., and Sixou, P., 1979, Molec. Phys., 38, 1275.

[23] Palmer, R. G., and Stein, D. L., 1984, Phys. Rev. Lett., 53, 958.

[24] Kohlraus, R., 1847, Ann. Phys., 12, 394.

[25] Wang, C. H., Ma, R. J., Fytas, G., and Dorfmüller, Th., 1983, J. chem. Phys., 78, 5863.

[26] Vogel, H., 1921, Phys. Z., 22, 645.

[27] Fulcher, G. S., 1925, J. Am. Ceram. Soc., 8, 339.

[28] More precisely, one should distinguish between the length over which the molecular orientations are correlated and the length over which the disclination lattice is correlated. 